\title{
Quantitative assessment of the response to therapy in achalasia of the cardia
}

\author{
C S ROBERTSON, J G HARDY, AND M ATKINSON \\ From the Departments of Surgery and Medical Physics, Queen's Medical Centre, Nottingham
}

SUMMARY Radionuclide oesophageal transit studies and manometry have been carried out in 15 patients with achalasia of the cardia, before treatment, after a course of nifedipine and after pneumatic bag dilatation. Transit studies were also done in 10 patients after cardiomyotomy and in 10 normal subjects. Images were recorded with the subjects seated in front of a gamma camera while swallowing a $10 \mathrm{ml}$ bolus of ${ }^{99} \mathrm{Tc}^{\mathrm{m}}$-tin colloid and then after a further drink of $50 \mathrm{ml}$ water. There was marked retention of tracer in the oesophagus in patients with achalasia compared with rapid clearance in control subjects. Bag dilatation significantly reduced lower oesophageal sphincter pressure but there was no significant difference in the $50 \%$ clearance time or percentage dose retained at $100 \mathrm{~s}$ before and after the treatments. Oesophageal clearance of tracer after the additional drink of water, was improved by bag dilatation. Oesophageal transit in the patients after cardiomyotomy was similar to that in patients who had undergone bag dilatation. There was considerable retention of the tracer in the oesophagus overnight, but this did not result in pulmonary aspiration. Radionuclide oesophageal transit studies provided a quantitative assessment of therapy in achalasia and the proportion of tracer retained after the additional drink proved to be a sensitive measure of response to treatment. Nifedipine proved ineffective as a treatment for achalasia. Bag dilatation and cardiomyotomy were of similar value.

The assessment of treatment in achalasia of the cardia is commonly based on symptoms and less frequently upon changes in the lower oesophageal sphincter pressure (LOSP) ${ }^{12}$ or the height of barium above the cardia on barium swallow. ${ }^{3}$ None of these tests, however, quantifies the functional disturbance of impaired emptying of the oesophagus or measures oesophageal food residue. Patients with a large food residue are considered to be at risk of pulmonary aspiration. Additionally, retention oesophagitis has been implicated in the aetiology of squamous carcinoma of the oesophagus complicating achalasia. ${ }^{4}$ Effective treatment should therefore relieve symptoms and also abolish or at least reduce any oesophageal residue.

Address for correspondence: Mr C S Robertson, FRCS, Dept of Surgery, Floor E, West Block, University Hospital, Queen's Medical Centre, Nottingham NG7 2UH.

Accepted for publication 7 November 1988.
Forceful dilatation of the cardia and Heller's cardiomyotomy remain the standard treatments for achalasia and debate continues regarding the relative merits of each. Interest in the medical treatment of achalasia has recently been revived, however, with the development of the calcium channel blocking drugs $^{5}$ which have been shown to reduce LOSP in healthy subjects and patients with achalasia. ${ }^{1267}$ Although they have been reported to relieve dysphagia in achalasia, little is known of their effect on the clearance of oesophageal food residues. ${ }^{184}$

In the present study radionuclide oesophageal transit studies have been undertaken to quantify oesophageal emptying in patients with achalasia, before treatment and after nifedipine therapy and pneumatic bag dilatation. For comparative purposes, measurements were made in healthy subjects and in a separate group of patients with achalasia who were asymptomatic after cardiomyotomy. 
Methods

\section{PATIENTS}

Fifteen patients (seven men) with clinically, radiologically and manometrically established achalasia of the cardia were studied. Their median age was 45 years $(20-63)$ and they had had dysphagia for a median of two years before treatment (three monthsfive years). All patients had a dilated oesophagus $(\geqslant 40 \mathrm{~mm}$ diameter) but only two had a markedly dilated, 'sigmoid', oesophagus.

A separate group of 10 patients (six men), having a median age of 35 years (13-59), was studied after Heller's cardiomyotomy for achalasia. The median interval since operation was three years (one to 14). None had undergone an antireflux procedure nor developed a peptic oesophageal stricture postoperatively. Only one had dysphagia at the time of study. Normal transit values were established with a control group of 10 healthy volunteers (five men) median age 29 years $(20-61)$.

The study was approved by the Hospital Ethical Committee and patients gave written, informed consent.

\section{OESOPHAGEAL TRANSIT STUDY}

The oesophageal transit studies were undertaken on fasted subjects seated upright in front of a gamma camera. The field of view of the camera covered the whole of the oesophagus and upper stomach. Ten millilitres of water containing $10 \mathrm{MBq}{ }^{99} \mathrm{Tc}^{\mathrm{m}}$-tin colloid was run into the mouth by syringe, and then swallowed as a bolus. Images of $0.5 \mathrm{~s}$ duration were recorded over two minutes during oesophageal transit of the tracer and the data stored by computer for subsequent analysis. At the end of the dynamic study the subjects drank a further $50 \mathrm{ml}$ water and a static image of $30 \mathrm{~s}$ duration was immediately recorded to assess clearance of residual tracer from the oesophagus.

The gamma camera images were displayed on a television monitor and a region of interest drawn over the whole of the oesophagus taking care to avoid the mouth and the stomach. A time activity curve was generated (Fig. 1) for the whole of the oesophagus. Three indices of transit were obtained: the time for $50 \%$ of the activity to be cleared from the oesophagus; the percentage of the activity retained in the oesophagus $100 \mathrm{~s}$ from the start of the study. In addition, the percentage of the dose retained in the oesophagus after the additional drink of $50 \mathrm{ml}$ water was recorded.

\section{OESOPHAGEAL MANOMETRY}

This was done using a triple lumen PVC catheter constantly perfused with water by a pneumohydraulic pump (Mui Scientific, Canada). The catheter was connected to electrically isolated pressure transducers and recordings were made using a multi-channel pen recorder. Pressure measurements were made at the upper and lower sphincter positions. Additionally the pressure and motility in the oesophageal body were assessed, using the station pull through technique. ${ }^{10}$
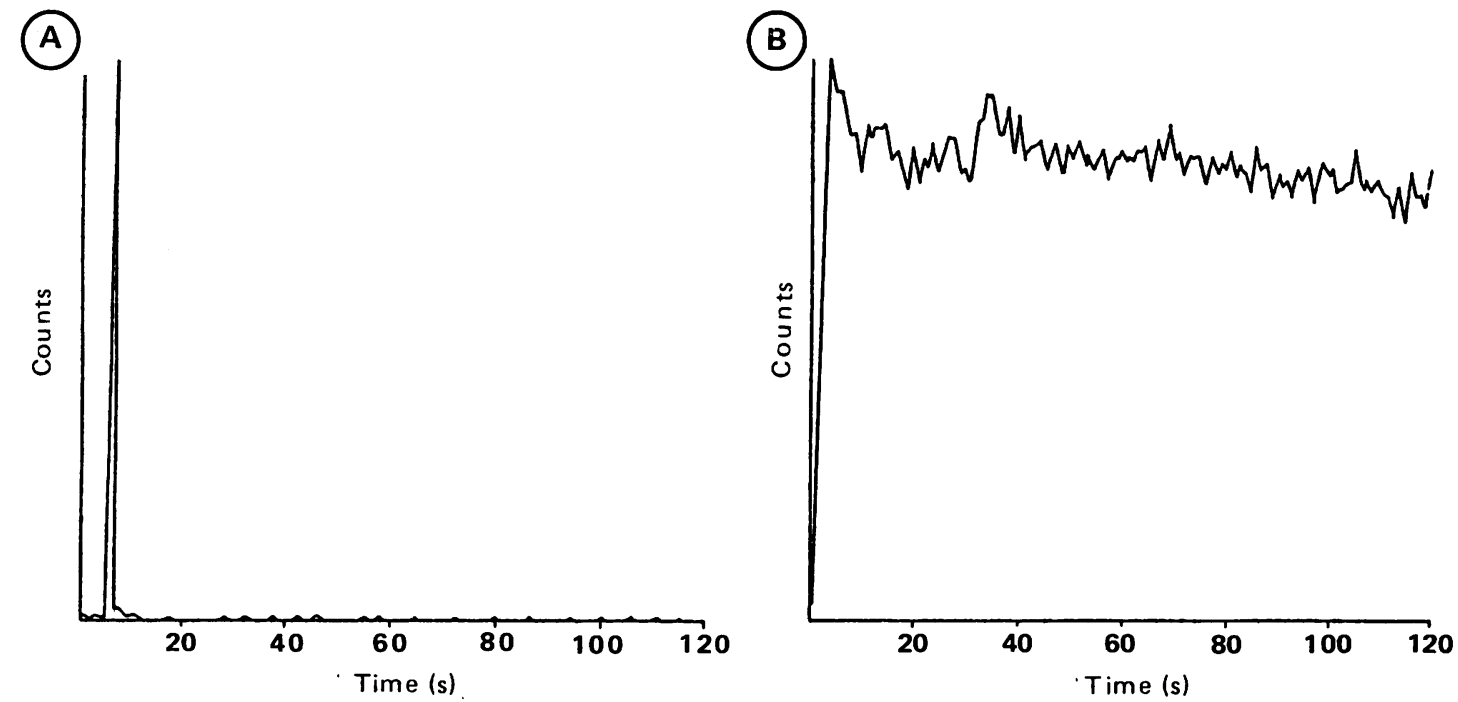

Fig. 1 Typical examples of time/activity curves for tracer in the oesophagus in: (a) healthy volunteer; (b) patient with achalasia of the cardia. 
Table 1 Dysphagia grading

$0=$ No dysphagia

$1=$ Dysphagia no more than once per week

$2=$ Dysphagia more than once per week

$3=$ Dysphagia with every meal

$4=$ Unable to finish meal because of dysphagia or regurgitating food or losing weight

STUDY PROTOCOL

After an initial oesophageal transit study (OTS) patients were treated with $10 \mathrm{mg}$ nifedipine capsules which were opened and the contents placed under the tongue 15 minutes before meals for one month. After this course of treatment the OTS and manometry were repeated 15 minutes after a dose of the drug. The patients subsequently underwent pneumatic bag dilatation of the cardia under general anaesthesia using a $4.5 \mathrm{~cm}$ diameter Rider-Moeller bag. This was positioned across the cardia over an endoscopically placed guide wire and inflated to a pressure of 300 mmHg for three minutes." The patients were reassessed by manometry and OTS one month after dilatation.

At each attendance the patients were weighed and had a symptomatic assessment of their dysphagia using the scale in Table 1. An assessment of oesophageal food residues was made at initial diagnostic oesophagoscopy and after nifedipine treatment immediately before bag dilatation. Patients were not re-endoscoped after bag dilatation. The results from these patients were compared with those from a separate group of 10 patients who had previously undergone cardiomyotomy.

Pulmonary aspiration of oesophageal contents was assessed in 10 of the 15 untreated achalasia patients. Immediately before retiring for the night the patients drank $10 \mathrm{ml}$ water radiolabelled with $10 \mathrm{MBq}{ }^{4 \%} \mathrm{Tc}^{\mathrm{m}}$ tin colloid. Before breakfast the following morning a

Table 2 Effect of various therapies on oesophageal emptying (median values)

\begin{tabular}{|c|c|c|c|}
\hline Subjects & $\begin{array}{l}\text { Time for } \\
50 \% \text { clear- } \\
\text { ance (s) }\end{array}$ & $\begin{array}{l}\text { Dose } \\
\text { retained at } \\
100 \mathrm{~s}(\%)\end{array}$ & $\begin{array}{l}\text { Proportion retained } \\
\text { after additional } \\
\text { drink }(\%)\end{array}$ \\
\hline $\begin{array}{l}\text { Control subjects } \\
\qquad(n=10)\end{array}$ & 5 & 3 & 0 \\
\hline \multicolumn{4}{|l|}{ Achalasia $(n=15)$} \\
\hline Pretreatment & $>100$ & 68 & 78 \\
\hline Post nifedipine & $>100$ & 72 & $81)_{*}$ \\
\hline Post bag diltn & $>100$ & 63 & $15\}^{*}$ \\
\hline \multicolumn{4}{|l|}{ Achalasia $(n=10)$} \\
\hline $\begin{array}{l}\text { Previous } \\
\text { cardiomyotomy }\end{array}$ & $>100$ & 75 & 11 \\
\hline
\end{tabular}

*Mann Whitney U-test, $U=34 ; p<0 \cdot 025$.

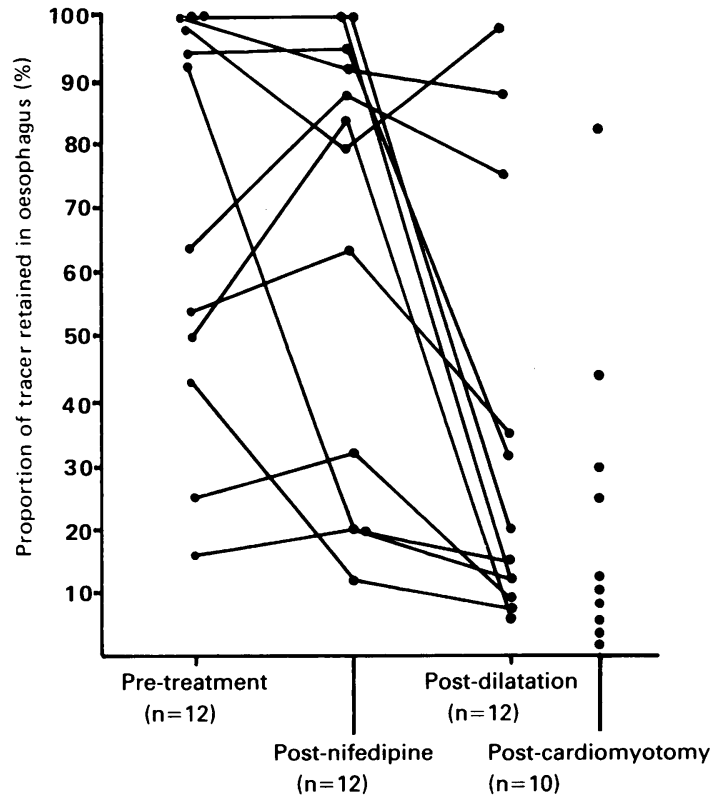

Fig. 2 Effect of the additional $50 \mathrm{ml}$ water on oesophageal retention of the tracer.

static image of the chest was recorded and an assessment made of tracer uptake within the lungs.

STATISTIC ANALYSIS

Data were analysed by Student's $t$ test and the MannWhitney U-test where appropriate. A value of $\mathrm{p}<0.05$ was taken to denote statistical significance.

\section{Results}

All 15 patients completed the study. The sublingual nifedipine was well tolerated with few side effects apart from mild headache reported by three patients. There were no complications as a result of dilatation.

OESOPHAGEAL TRANSIT STUDIES

The time activity curve for a healthy volunteer is shown in Figure 1a. This is characterised by the sharp rise and fall in count rate as the bolus of tracer passed rapidly through the oesophagus. The median $50 \%$ clearance time was five seconds with minimal retention of the dose at 100 seconds from the start of the study (Table 2). All the tracer was cleared from the oesophagus in the healthy subjects after an additional drink of $50 \mathrm{ml}$ water. The patients with achalasia, however, showed a different but typical time activity curve (Fig. 1b). This was characterised by a sharp rise in activity as the tracer entered the oesophagus but the activity plateaued as little progressed into the stomach. The quantitative results in the achalasia 
Table 3 Effect of various therapies on symptoms, weight gain, and lower oesophageal sphincter pressure

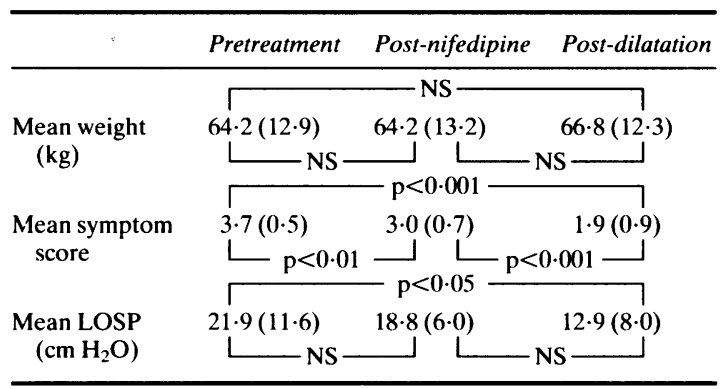

Mean (SD): Student's $t$ test.

patients before and after the treatments are shown in Table 2. Before treatment, after nifedipine and bag dilatation, the $50 \%$ clearance time remained in excess of 100 seconds and on average approximately $70 \%$ of the dose was retained in the oesophagus at 100 seconds. Overall, after the additional $50 \mathrm{ml}$ water oesophageal emptying was improved only after bag dilatation (Table 2). The effect of the additional drink of water in the three phases of the study is plotted for individual patients in Figure 2. Complete data were obtained for only 12 of the 15 patients as a result of computer malfunction. Three patients had a poor emptying response to bag dilatation and in only one of these did LOSP show a significant fall indicating that a further dilatation or cardiomyotomy may be necessary. The results in the patients after cardiomyotomy were comparable with those obtained after bag dilatation (Fig. 2).

MANOMETRY

Nifedipine had no significant effect on mean LOSP (Table 3), but after bag dilatation there was a significant fall in mean LOSP compared with pretreatment values. There was no close relationship between change in LOSP and change in oesophageal emptying after treatment. Neither nifedipine nor bag dilatation had any effect on lower oesophageal sphincter relaxation, motility in the oesophageal body or upper oesophageal sphincter function.

\section{CLINICAL ASSESSMENT}

No patients' symptoms worsened while taking nifedipine and there was a slight improvement in their mean symptom score (Table 3). Ten of 15 patients reported an improvement in symptoms on nifedipine but grade 2 dysphagia was the best result obtained and this in only three patients. After bag dilatation there was a significant improvement in mean symptom score and in none of the patients did symptoms deteriorate. Twelve of the 15 patients

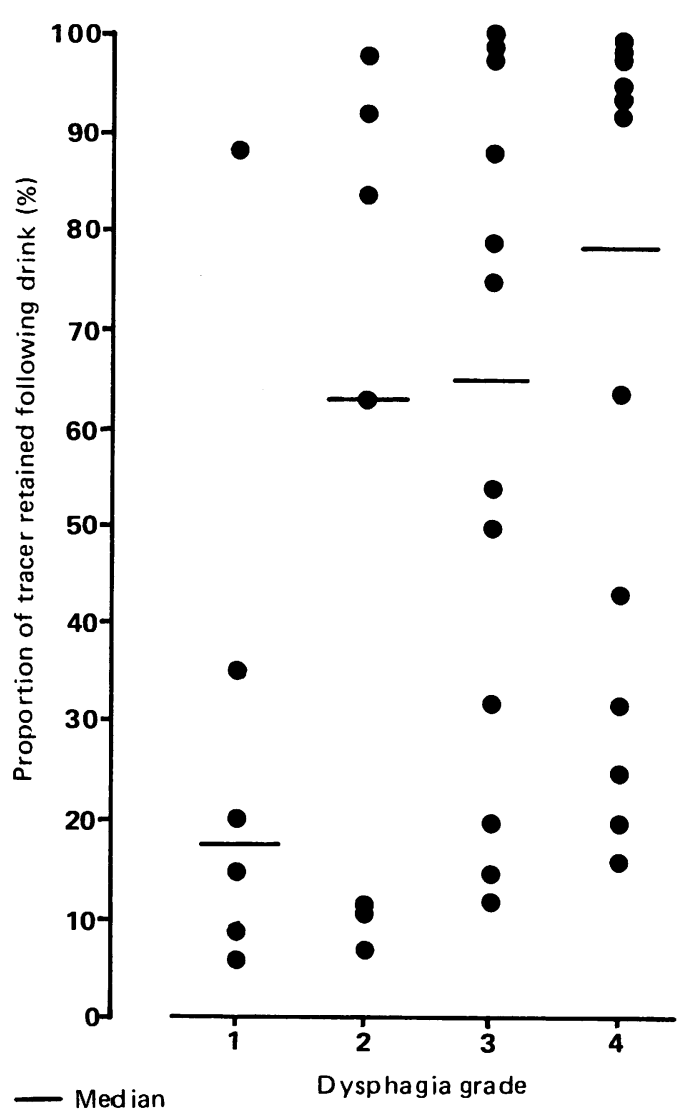

Fig. 3 Comparison of symptomatic response and retention of tracer after $50 \mathrm{ml}$ water in all patients at each stage of the study.

reported an improvement in symptoms, with five achieving grade 1 dysphagia. No patient became completely dysphagia free, however, after either treatment.

A comparison of dysphagia grade and retention of radiolabelled tracer after the additional drink, irrespective of treatment stage is shown in Figure 3. The median tracer retention is lowest in the grade 1 group. For the other groups, however, there is a very wide spread of retention with little difference between the median values. The two patients who had a sigmoid oesophagus exhibited results similar to those obtained from the other patients.

Overall, there was no significant increase in the patients' weights during the study. The study period was, however, relatively short and none of the patients was particularly malnourished. All patients had an oesophageal food residue at endoscopy before nifedipine therapy and in only one patient was no residue seen after the drug treatment. 
ASPIRATION STUDIES

All 10 patients exhibited marked tracer retention in the oesophagus the morning after the overnight study. In none of the patients, however, was any tracer detected in the lungs.

\section{Discussion}

Radionuclide imaging provides a simple, physiological procedure for assessing oesophageal transit. It is generally recommended ${ }^{12}$ that studies should be carried out supine, so as to reduce the influence of gravity and thereby enhance the sensitivity for the detection of abnormal peristalsis and minor impairment of oesophageal emptying. In achalasia, peristalsis in the smooth muscled part of the oesophagus is usually unrecordable and oesophageal emptying is dependent solely upon gravity. Therefore whilst studies in the supine position are better for diagnosis, those in the upright posture are more appropriate for the assessment of the effects of treatment in achalasia. There was marked retention of the $10 \mathrm{ml}$ bolus of tracer before and after all the treatments. Thus swallowing a small volume of liquid provided a sensitive test for the presence of achalasia. The additional $50 \mathrm{ml}$ drink of water gave a good indication of the response to treatment.

None of the patients complained of chest symptoms and although all 10 patients in the aspiration study had a large residue of tracer in the oesophagus overnight, none showed evidence of pulmonary aspiration. In all patients upper oesophageal sphincter function was normal and presumably adequate to prevent the passage of oesophageal contents into the pharynx.

Patients were initially treated with nifedipine as this offered the potential of a non-invasive therapy. This drug has been shown in clinicals trials, involving healthy subjects and patients with achaiasia, to reduce LOSP. ${ }^{1267}$ This finding was not confirmed, however, in the present study. An alternative drug, isosorbide dinitrate, has been reported ${ }^{7.3}$ to be effective in relieving symptoms, reducing LOSP and enhancing radionuclide oesophageal emptying in patients with achalasia, but it is associated with over $50 \%$ failure rate with unpleasant side effects.

From the results of the present study it is apparent that little change in the LOSP followed nifedipine in a $10 \mathrm{mg}$ dosage and there was little improvement in swallowing of even liquids in most patients. Three patients showed poor clearance throughout all stages of assessment (Fig. 2). For these patients, even after bag dilatation, over $70 \%$ of the tracer was retained after the additional drink of water. This objective measure can be regarded as evidence of a poor response to treatment. Interestingly, the subjective scores for these patients after treatment showed changes from initial values of 3,4 , and 4 to 1,2 , and 3 respectively, thus confirming the difficulty of clinical evaluation based solely on symptomatic response. Although overall there was a significant reduction in LOSP after bag dilatation (Table 3 ) this did not correlate closely with either relief of symptoms or improvement in emptying. This poor correlation between symptomatic and objective improvement has been noted previously ${ }^{7}$ and presumably represents a placebo effect. The present findings indicate that symptomatic improvement after sublingual nifedipine in a dosage of $10 \mathrm{mg}$ before meals, is considerably less than after pneumatic bag dilatation. This, together with the absence of objective evidence of improvement in oesophageal emptying, suggests that the drug has little place in the treatment of achalasia.

In terms of symptomatic improvement and oesophageal emptying, pneumatic bag dilatation was clearly superior to nifedipine in the treatment of achalasia. Although oesophageal emptying after the $50 \mathrm{ml}$ water swallow in the upright position did not reach normality it was greatly improved by pneumatic bag dilatation and approximated to the emptying rate found after successful cardiomyotomy. Obviously, short term measurements such as these cannot form a valid basis for assessment of long term outcome. Further studies are needed to establish the duration of improvement in oesophageal emptying after pneumatic bag dilatation.

\section{References}

1 Bortolotti M, Labo G. Clinical and manometric effects of nifedipine in patients with esophageal achalasia. Gastroenterology 1981; 80: 39-44.

2 Blackwell JN, Holt S, Heading RC. Effect of nifedipine on oesophaeal motility and gastric emptying. Digestion 1981; 21: 50-6.

3 Cohen N. An end point for pneumatic dilatation of achalasia. Gastrointest Endosc 1975; 22: 29.

4 Harley HRS. Achalasia of the cardia. Bristol: Wright, 1978: 106.

5 Cohen S. Esophageal motility disorders and their response to calcium channel antagonists. Gastroenterology 1987; 93: 201-3.

6 Hongo M, Traube M, McAllister RG, McCallum RW. Effects of nifedipine on esophageal motor function in humans: correlation with plasma nifedipine concentration. Gastroenterology 1984; 86: 8-12.

7 Gelfond M, Rozen P, Gilat T. Isosorbide dinitrate and nifedipine treatment of achalasia: a clinical, manometric and radionuclide evaluation. Gastroenterology 1982; 83: 963-9.

8 Traube $M$, Hongo $M$, Magyar L, McCallum RW. Effects of nifedipine in achalasia and in patients with 
high-amplitude peristaltic esophageal contractions. JAMA 1984; 252: 1733-6.

9 Traube M, DuBovik S, Magyar L, McCallum RW. Sublingual nifedipine in achalasia; a randomised double blind study. Gastroenterology 1986; 90: 1670.

10 Dodds WJ. Instrumentation and methods for intraluminal esophageal manometry. Arch Intern Med 1976; 136: $515-23$.
11 Fellows IW, Ogilvic AL, Atkinson M. Pneumatic dilatation in achalasia. Gut 1983; 24: 1020-3.

12 Russell COH, Hill LD, Holmes ER, Hull DA, Gannon R, Pope CE. Radionuclide transit: a sensitive screening test for esophageal dysfunction. Gastroenterology 1981; 80: 887-92.

13 Gelfond M, Rozen P, Keren S, Gilat T. Effect of nitrates on LOS pressure in achalasia: a potential therapeutic aid. Gut 1987; 22: 312-8. 